\title{
Robust Multiuser Detection in Synchronous DS-CDMA System with MRC Receive Diversity over Nakagami- $m$ Fading Channel
}

\author{
Srinivasa Rao $\mathrm{V}^{1, \mathrm{a}}$, Vinay Kumar $\mathrm{P}^{2, \mathrm{~b}}$, \\ Balaji $\mathrm{S}^{3, \mathrm{c}}$, Habibulla Khan ${ }^{3, \mathrm{~d}}$ and Anil Kumar $\mathrm{T}^{4, \mathrm{e}}$ \\ ${ }^{1}$ Department of ECE, Anurag Engineering College, Kodad, India 508206 \\ ${ }^{2}$ Department of ECE, MIC College of Technology, Kanchikacherla, India 521180 \\ ${ }^{3}$ Department of ECE, KL University, Vaddeswaram, India 522502 \\ ${ }^{4}$ Department of ECE, TRR Engineering College, Patancheru, India 502319 \\ avempatisr@ieee.org, ${ }^{b}$ pamulavk@ieee.org, ${ }^{2}$ tvakumar2000@yahoo.co.in
} Keywords: Influence function, maximal ratio combiner, $M$-estimator, Nakagami- $m$ distribution,
probability of error.

\begin{abstract}
This paper presents the robust multiuser detection in synchronous direct sequence-code division multiple access (DS-CDMA) systems with Maximal Ratio Combiner (MRC) receive diversity over frequency-nonselective, slowly fading Nakagami- $m$ channels in a non-Gaussian environment. Average probability of error is derived for decorrelating detector over single path Nakagami- $m$ fading channel. A new $M$-estimator proposed to robustify the detector is studied and analyzed. Simulation results show that the new $M$-estimator outperforms linear decorrelating detector, the Huber, and the Hampel estimator based detectors.
\end{abstract}

\section{Introduction}

Direct sequence-code division multiple access (DS-CDMA), a multiple access technique, has attracted considerable attention of researchers and used in many cellular mobile communication systems. Recent research has explored the potential benefits of optimum multiuser detection (MUD) techniques to combat multiple access interference (MAI). Complexity of optimum multiuser detector, based on maximum likelihood (ML) criterion, grows exponentially with number of active users [1]. Suboptimal multiuser detectors are the alternatives with identical perfornance. The decorrelating detector presented in [2] is used to overcome the near-far problem as well as MAI. This decorrelator requires no knowledge of received signal amplitudes but it requires matrix inversion, which increases the computational complexity.

The performance of any wireless communication system degrades with interference and multipath fading. It is possible to mitigate the effects of multipath fading by employing diversity combining techniques [3]. In diversity combing, several copies of information-bearing signal are combined to increase the signal-to-noise ratio (SNR) [4]. Selection combining (SC), maximal ratio combining (MRC), equal gain combining (EGC), switch and stay combining (SSC) and threshold combining (TC) are the commonly employed linear receive diversity combining techniques $[3,5]$. MRC, EGC and SC are studied and implemented widely so that they can be considered as three basic diversity combining techniques [6].

In the literature, the Nakagami- $m$ distribution received considerable attention as it can provide a good fit to measured data in different fading environments [7] like Rayleigh, log-normal or Ricean fading channels. It can also accommodate fading conditions that are more or less severe than that of the Rayeigh fading channel. Nakagami- $m$ fading is often encountered in many practical applications such as cellular mobile communications [8]. Recently, Beaulieu et. al [6] considered Nakagami-0.5 distribution as a special case of the Nakagami- $m$ distribution with $m=0.5$ and proved that a $D$-branch MRC diversity system with Nakagami- 0.5 fading is equivalent to a single branch Nakagami- $D / 2$ system with $D$ times greater power. Nakagami- 0.5 fading model will have great theoretical interest as a limiting worst case with severe fading and as the one-sided Gaussian case [6]. Performance 
analysis of MUD in synchronous DS-CDMA communication system using decorrelating and decision feedback (DFB) detectors with single path Nakagami fading is presented in [9] by deriving the lower bounds of the probability of error. Recently, [10] analyzed decorrelator receiver for DS-CDMA mobile radio system, employing RAKE reception and both MRC and SC diversity, through Nakagami fading channel. Performance of digital cellular mobile radio systems in a frequency non-selective Rayleigh fading channel with lognormal shadowing, including the effects of white Gaussian noise, narrow-band impulsive noise interference and co-channel interference is presented by [11].

There exist in the literature a number of different approaches to the robust estimation problem, and the $M$-estimator is one of the most sophisticated approaches to this problem. Recently, the problem of robust multiuser detection in non-Gaussian channels has been addressed in the literature [12], which was developed based on the Huber and the Hampel $M$-estimators, respectively. This paper presents the performance of a DS-CDMA system with MRC receive diversity scheme over frequency-nonselective, slowly fading Nakagami- $m$ multipath channel in a non-Gaussian environment. An expression for average probability of error of a decorrelating detector is derived. This expression is used to compute the probability of error of linear decorrelating detector, the Huber and the Hampel estimator based detectors, and the proposed $M$-estimator.

The remaining portion of the paper is organized as follows: Synchronous DS-CDMA system over multipath fading channel in non-Gaussian environment is considered in Section II. $M$-estimation based regression is presented in Section III. The asymptotic performance of $M$-decorrelator is discussed in Section IV, by deriving probability of error. Section V discusses simulation results and finally, conclusion is drawn in Section VI.

\section{System and Channel Model}

a. Synchronous DS-CDMA system. This paper considers an $L$ - user synchronous DS-CDMA system signaling through frequency-nonselective, slowly fading channels. The received signal during $i^{\text {th }}$ symbol interval is given by [9]

$$
r(t)=\sum_{i=0}^{\infty} \sum_{l=1}^{L} \sqrt{\frac{2 E_{b_{l}}}{T}} \alpha_{l}(i) \mathrm{e}^{-j \theta_{l}(i)} b_{l}(i) s_{l}(t-i T)+n(t),
$$

where $\alpha_{l}[i]$ is the fading gain of the $l^{\text {th }}$ user's channel during the $i^{\text {th }}$ symbol interval, $b_{l}[i]$ is the $i^{\text {th }}$ bit of the $l^{\text {th }}$ user, $s_{l}(t), \int_{0}^{T} s_{l}^{2}(t) d t=1$, is the spreading waveform of the $l^{\text {th }}$ user and $n(t)$ is assumed as a zero-mean complex non-Gaussian noise. The probability density function of this noise model has the form [12]

$$
f=(1-\varepsilon) N\left(0, v^{2}\right)+\varepsilon N\left(0, \kappa v^{2}\right)
$$

with $v>0,0 \leq \varepsilon \leq 1$, and $\kappa \geq 1$. Here $N\left(0, v^{2}\right)$ represents the nominal background noise and the $N\left(0, \kappa v^{2}\right)$ represents an impulsive component, with $\varepsilon$ representing the probability that impulses occur. It is assumed that the $l^{\text {th }}$ user employs binary phase shift keying (BPSK) modulation to transmit the data bits $b_{l} \in[-1,1]$ with equal probability and a symbol rate $1 / T$. Here, it is also assumed that the signal of each user arrives at the receiver through an independent, single-path fading channel.

The received signal $r(t)$ is passed through a matched filter bank and its output at the $i^{\text {th }}$ sampling instant can be represented as a column vector of length $L$ as

$$
\mathbf{r}[i]=\mathbf{R W}[i] \mathbf{b}[i]+\mathbf{n}[i]
$$


where $\mathbf{R}$ is the signature cross-correlation matrix with elements $\rho_{l m}=\int_{0}^{T} s_{l}(t) s_{m}(t) d t$, $(l, m=1,2, \ldots L)$, with unity diagonal elements, $\mathbf{b}$ is the data vector with components $b_{l}$, and the vector $\mathbf{n}$ contains the corresponding samples of the noise process. The channel matrix $\mathbf{W}[i]$ is the diagonal matrix with diagonal elements $W_{l, l}=\sqrt{E_{b_{l}}} C_{l}(i)>0$ with $C_{l}(i)=\alpha_{l}(i) \mathrm{e}^{-j \theta_{l}(i)}$. When the channel is assumed as a slowly fading channel, $C_{l}(i)$ can be modeled as a constant over a symbol period $T$, and the phase $\theta_{l}(i)$ can be estimated from the received signal.

b. Nakagami-m fading channel. Assuming the Nakagami- $m$ fading channel, $\alpha_{l}[i]$ are independent and identically distributed Nakagami random variables with probability density function (PDF) given by

$$
p\left(\alpha_{l}\right)=2\left(\frac{m}{\Omega}\right)^{m D} \frac{\alpha_{l}^{2 m D-1}}{\Gamma(m D)} \exp \left(-\frac{m}{\Omega} \alpha_{l}^{2}\right)
$$

where, $m$ is the Nakagami fading parameter that determines the severity of the fading, $\Omega=E\left[\alpha_{l}^{2}\right]$ is the average channel power, $\mathrm{E}[\cdot]$ is statistical expectation and $\Gamma(\cdot)$ is the complete Gamma function. In a wireless mobile channel, the multipath intensity profile follows the relation [13]

$$
\Omega=\Omega_{\mathrm{O}} \exp (-\delta),
$$

where $\Omega_{\mathrm{o}}$ is the initial path strength and $\delta$ is the power decay factor.

Consider a $D$ - branch diversity receiver in slowly fading Nakagami- $m$ channels with SNR per bit on $l^{\text {th }}$ branch as $\gamma_{l}, l=1,2, \ldots, D$. The overall instantaneous SNR per bit at the output of MRC over Nakagami- $m$ fading channels is given by [13]

$$
\gamma_{b}=\frac{E_{b_{l}}}{N_{o}} \sum_{l=1}^{D} \alpha_{l}^{2}=\sum_{l=1}^{D} \gamma_{l}
$$

where, $\gamma_{l}=\left(E_{b_{l}} / N_{o}\right) \alpha_{l}^{2}, E_{b_{l}}$ is the transmitted signal energy per bit of $l^{\text {th }}$ user and $N_{o} / 2$ is the power spectral density of noise. The output SNR per bit $\gamma_{l}$ is gamma distributed with PDF

$$
p\left(\gamma_{l}\right)=\left(\frac{m}{\bar{\gamma}_{l}}\right)^{m D} \frac{\gamma_{l}^{m D-1}}{\Gamma(m D)} \exp \left(-m \frac{\gamma_{l}}{\bar{\gamma}_{l}}\right), \quad m \geq 0.5
$$

where $\bar{\gamma}_{l}=\left(E_{b_{l}} / N_{o}\right) E\left[\alpha_{l}^{2}\right] \geq 0$ is the average output received SNR per bit for a channel.

\section{M-Estimation}

In $M$-estimates, unknown parameters $\theta_{1}, \theta_{2}, \ldots \theta_{L}$ (where $\theta=A b$ ) are solved by minimizing a sum of function $\rho(\cdot)$ of the residuals

$$
\hat{\boldsymbol{\theta}}=\underset{\boldsymbol{\theta} \in \mathfrak{R}^{L}}{\arg \min } \sum_{j=1}^{N} \rho\left(r_{j}-\sum_{l=1}^{L} s_{j}^{l} \theta_{l}\right),
$$


where $\rho$ is a symmetric, positive-definite function with a unique minimum at zero, and is chosen to be less increasing than square and $N$ is the processing gain. Suppose that $\rho$ has a derivative with respect to the unknown parameters $\theta\left(\psi=\rho^{\prime}\right)$, called the influence function, since it describes the influence of measurement errors on solutions. The solution to Eq. (8) satisfies the implicit equation [12]

$$
\sum_{j=1}^{N} \psi\left(r_{j}-\sum_{l=1}^{L} s_{j}^{l} \theta_{l}\right) s_{j}^{k}=0, \quad k=1, \ldots, L
$$

or in vector form

$$
\mathbf{S}^{T} \psi(\mathbf{r}-\mathbf{S} \boldsymbol{\theta})=\mathbf{0}_{L},
$$

where $\mathbf{S}^{T}$ is the transpose of $\mathbf{S}$ and $\mathbf{0}_{L}$ is an all zero vector of length $L$. Eq. (8) is called an $M$-estimator. Different influence functions yield solutions with different robustness properties. Therefore, an influence function $\psi(\cdot)$ should be chosen such that it yields a solution that is not sensitive to outlying measurements.

\section{a. Influence functions}

$M$-estimators are generalizations of the usual maximum likelihood estimates. In this section, the influence functions of $M$-estimators proposed in the literature $[12,14]$ are listed (see Fig. 1).

Huber's estimator. The Huber's $M$-estimator is determined by the Huber penalty function $\rho_{H U}(\cdot)$ and its derivative given by

$$
\psi_{H U}(x)=\left\{\begin{array}{lll}
\frac{x}{v^{2}} & \text { for } & |x| \leq k v^{2} \\
k \operatorname{sgn}(x) & \text { for } & |x|>k v^{2}
\end{array} .\right.
$$

Hampel's estimator. Similarly, the Hampel's $M$-estimator is determined by the Hampel's penalty function $\rho_{H A}(\cdot)$ and its derivative given by

$$
\psi_{H A}(x)=\left\{\begin{array}{crl}
x & \text { for } & |x| \leq a \\
a \operatorname{sgn}(x) & \text { for } & a<|x| \leq b \\
a \operatorname{sgn}(x) \frac{c-|x|}{c-b} & \text { for } & b<|x| \leq c \\
0 & \text { for } & |x|>c
\end{array} .\right.
$$

Proposed estimator. The proposed $M$-estimator is given by

$$
\psi_{P R O}(x)=\left\{\begin{array}{cc}
x & \text { for }|x| \leq a \\
a \operatorname{sgn}(x) & \text { for } a<|x| \leq b \\
\frac{a}{b} x \exp \left(1-\frac{x^{2}}{b^{2}}\right) & \text { for }|x|>b
\end{array} .\right.
$$


The choice of the constants $a\left(=k v^{2}\right)$ and $b\left(=2 k v^{2}\right)$ depends on the robustness measures derived from the influence function of [14], where $k$ is a constant.

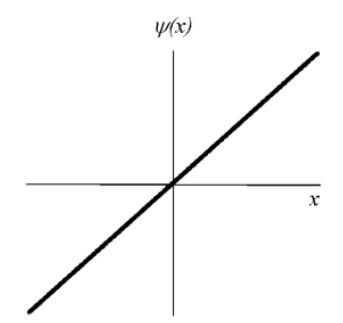

(a)

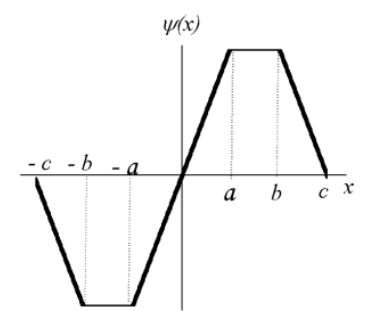

(c)



(b)

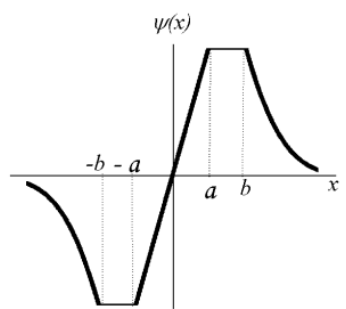

(d)

Figure 1. Influence functions of (a) the linear decorrelating detector, (b) Huber estimator, (c) Hampel estimator, and (d) the proposed estimator.

\section{Asymptotic Performance of $M$-Decorrelator}

Asymptotic probability of error for the class of decorrelating detectors described by (9) for large processing gain $N$, is given by [12]

$$
P_{e}^{l} \equiv \operatorname{Pr}\left(\hat{\theta}_{l}<0 \mid \theta_{l}>0\right)=Q\left(\frac{W_{l}}{v \sqrt{\left[\mathbf{R}^{-1}\right]}}\right)
$$

where $Q(x)$ is Gaussian $Q$ - function defined by $Q(x)=\int_{x}^{\infty} \frac{1}{\sqrt{2 \pi}} \exp \left(-\xi^{2} / 2\right) d \xi, x \geq 0$, and

$$
v^{2}=\frac{\int \psi^{2}(u) f(u) d u}{\left[\int \psi^{\prime}(u) f(u) d u\right]^{2}} .
$$

Over Nakagami- $m$ flat fading channel, $W_{l}$ is a Nakagami random variable. By assuming $\alpha_{l}=\left|W_{l}\right|$, the probability of error, (14), for user \#1 can be expressed, using the relation between $Q(\cdot)$ and complementary error function $\operatorname{erfc}(\cdot)$, as [9]

$$
P_{e}^{1}=Q\left(\frac{\alpha_{1}}{v \sqrt{\left[\mathbf{R}^{-1}\right]_{11}}}\right)=\frac{1}{2} \operatorname{erfc}\left(\frac{\alpha_{1}}{v \sqrt{2\left[\mathbf{R}^{-1}\right]_{11}}}\right) .
$$

The average probability of error for decorrelating detector over single path Nakagami- $m$ fading channel is given by [10] 


$$
\overline{P_{e}^{1}}=\int_{0}^{\infty} P_{e}\left(\alpha_{1}\right) p\left(\alpha_{1}\right) d \alpha_{1}
$$

Using Eq. (4) and Eq. (16), Eq. (17) can be expressed as

$$
\overline{P_{e}^{1}}=\left(\frac{m}{\Omega}\right)^{m D} \frac{1}{\Gamma(m D)} \underbrace{\int_{0}^{\infty} \alpha_{1}^{2 m D-1} e^{-\frac{m}{\Omega} \alpha_{1}^{2}} \operatorname{erfc}\left(\frac{\alpha_{1}}{v \sqrt{2\left[\mathbf{R}^{-1}\right]_{11}}}\right) d \alpha_{1}}_{I_{1}} .
$$

Substituting $\xi^{2}=\frac{m}{\Omega} \alpha_{1}^{2}$ in the integral $I_{1}$ of $(18)$, we get

$$
\overline{P_{e}^{1}}=\left(\frac{\Omega}{2 m}\right)^{m D} \int_{0}^{\infty} \xi^{2 m D-1} e^{-\xi^{2} / 2} \operatorname{erfc}\left(\frac{\xi}{\sigma \sqrt{2}}\right) d \xi
$$

where $\sigma=\sqrt{\frac{2 m}{\Omega}\left[\mathbf{R}^{-1}\right]_{11}}$. From the properties of $\operatorname{erfc}(\cdot)$, integral in (19) can be expressed, for integer values of $m D$, as

$$
\begin{aligned}
& \int_{0}^{\infty} \xi^{2 m D-1} e^{-\xi^{2} / 2} \operatorname{erfc}\left(\frac{\xi}{\sigma \sqrt{2}}\right) d \xi \\
& =(m D-1) !\left[1-\left(\sigma^{2}+1\right)^{-1 / 2}\right]^{m D} \cdot \sum_{j}^{m D-1} 2^{-j}\left(\begin{array}{c}
m D-1+j \\
j
\end{array}\right) \cdot\left[1+\left(\sigma^{2}+1\right)^{-1 / 2}\right]^{j}
\end{aligned}
$$

Therefore, the average probability of error of the decorrelating detector can be obtained as

$$
\overline{P_{e}^{1}}=\left(\frac{1}{2}\right)^{m D} F^{m D} \cdot \sum_{j=0}^{m D-1} 2^{-j}\left(\begin{array}{c}
m D-1+j \\
j
\end{array}\right) \cdot G^{j},
$$

where $F=1-\left(\sigma^{2}+1\right)^{-1 / 2}$ and $G=1+\left(\sigma^{2}+1\right)^{-1 / 2}$.

\section{Simulation Results}

In this section, simulation results for the average probability of error are presented by computing Eq. (21) for different values of Nakagami fading parameter and for different order of diversity with $\Omega_{0}=10 \mathrm{~dB}, \delta=0.1,0.3$ and 0.9 .

Performance of decorrelating detector with different influence functions is shown in Fig. 2, Fig. 3, Fig. 4 and Fig. 5. In Fig. 2 and Fig. 3, the performance of four decorrelating detectors is studied by plotting the average probability of error versus the signal-to-noise ratio (SNR) corresponding to the user \#1 under perfect power control of a synchronous system with six users $(L=6)$ and a processing gain of $31(N=31)$. The noise distribution parameters are $\varepsilon=0.01 \& \kappa=100$. Similarly, in Fig. 4 and Fig. 5, performance is studied for noise distribution parameters $\varepsilon=0.1 \& \kappa=100$. The simulation results reveal the effect of Nakagami fading parameter and power decay factor. However, the proposed $M$-estimator outperforms the linear decorrelating detector and minimax decorrelating detector (both with Huber and Hampel estimators), even in highly impulsive noise. Moreover, this performance gain increases as the SNR increases. 




Fig. 2. Average probability of error versus SNR for user \#1 for linear multiuser detector (LS), minimax detector with Huber (HU), Hampel (HA) and proposed (PRO) $M$-estimator in synchronous CDMA channel with impulse noise, $\mathrm{N}=31, \varepsilon=$ $0.01 ; m=1, D=1 ; \Omega_{0}=10 \mathrm{~dB}, \delta=0,0.9$.

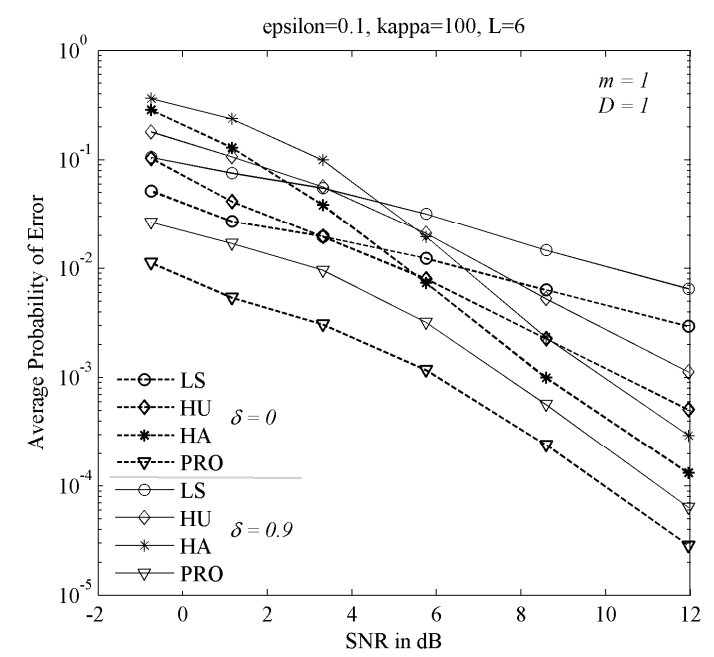

Fig. 4. Average probability of error versus SNR for user \#1 for linear multiuser detector (LS), minimax detector with Huber (HU), Hampel (HA) and proposed (PRO) $M$-estimator in synchronous CDMA channel with impulse noise, $\mathrm{N}=31, \varepsilon=$ $0.1 ; m=1, D=1 ; \Omega_{\mathrm{o}}=10 \mathrm{~dB}, \delta=0,0.9$.

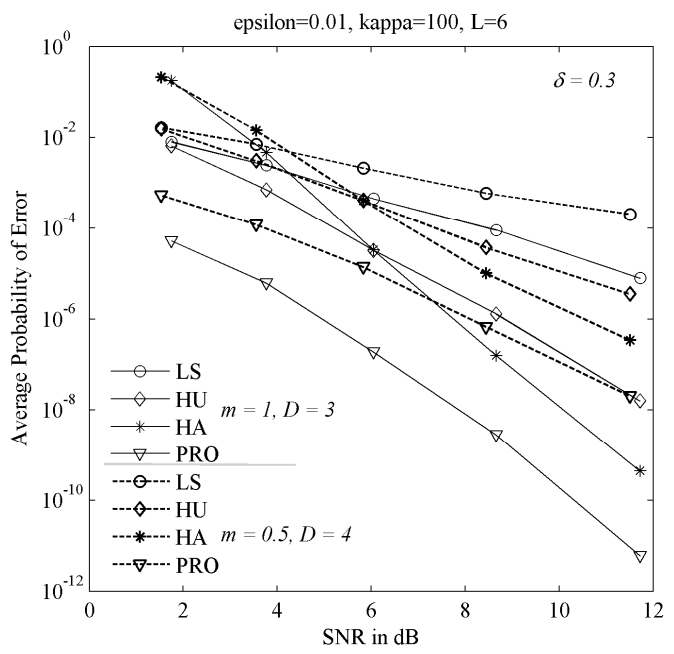

Fig. 3. Average probability of error versus SNR for user \#1 for linear multiuser detector (LS), minimax detector with Huber (HU), Hampel (HA) and proposed (PRO) $M$-estimator in synchronous CDMA channel with impulse noise, $\mathrm{N}=31, \varepsilon=$ $0.01 ; m=0.5,1 ; D=3,4 ; \Omega_{0}=10 \mathrm{~dB}, \delta=0.3$.

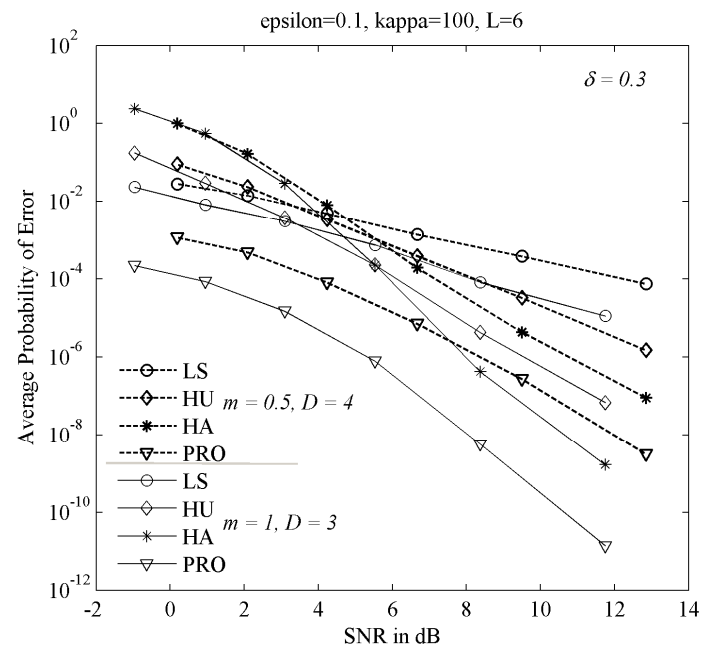

Fig. 5. Average probability of error versus SNR for user \#1 for linear multiuser detector (LS), minimax detector with Huber (HU), Hampel (HA) and proposed (PRO) $M$-estimator in synchronous CDMA channel with impulse noise, $\mathrm{N}=31, \varepsilon=$ $0.1 ; m=0.5,1 ; D=3,4 ; \Omega_{\mathrm{o}}=10 \mathrm{~dB}, \delta=0.3$.

\section{Concluding Remarks}

In this paper, robust multiuser detection in synchronous direct sequence (DS) code division multiple access (CDMA) system with Maximal Ratio Combiner (MRC) receive diversity over frequency-nonselective, slowly fading Nakagami- $m$ channels in a non-Gaussian environment is presented. An expression for average probability of error of the decorrelating detector is derived for integer values of $m D$. A new $M$-estimator based robust multiuser detection technique is proposed, which significantly outperforms the linear decorrelating detector and minimax robust multiuser detector (with Huber and Hampel $M$-estimators) in non-Gaussian ambient noise. 
Simulation results show that the proposed robust multiuser detector offers significant performance gain over the linear multiuser detector and the minimax decorrelating detectors with Huber and Hampel $M$-estimator, in non-Gaussian noise with little attendant increase in the computational complexity. Effect of fading parameter, diversity order and power decay factor on the performance of decorrelator is also studied.

\section{References}

[1] S. Verdu, "Computational complixity of optimum multiuser detection ," Algorithmica, pp. 303-312, May (1989).

[2] R. Lupas and S. Verdu, "Near-far resistance of multiuser detectors in asynchronous channels", IEEE Transacetions on Communications, Vol. 38, No. 4, pp. 496-508, April (1990).

[3] A. Goldsmith, Wireless Communications, Cambridge University Press,( 2005).

[4] G. Femenias, "MGF-based performance analysis of selection diversity with switching constraints in nakagami fading," IEEE Transactions on Wireless Communications, vol.5, no.9, pp.2328-2333, (2006).

[5] M. K. Simon, M.S. Alouini, Digital Communications Over Fading Channels, John Wiley \& Sons, (2000).

[6] N.C. Beaulieu and A.M. Rabiei, "Linear Diversity Combining on Nakagami-0.5 Fading Channels, " IEEE Transactions on Communications, vol. 59, no. 10, pp. 2742-2752, (2011).

[7] V.A. Aalo, G.P. Efthymoglou, "On the MGF and BER of Linear Diversity Schemes in Nakagami Fading Channels with Arbitrary Parameters," IEEE 69th Vehicular Technology Conference, vol., no., pp.1-5, 26-29 (2009).

[8] _ Mobile and Wireless Communications Physical Layer Development and Implementation, pp.57-76, InTech, (2010).

[9] Novakovic D. M., Dukic, M.L, "Multiuser detection analysis in DS- CDMA communication systems with Nakagami fading channels," IEEE $5^{\text {th }}$ International Symposium on Spread Sprectum Techniques and Applications, vol.3, no., pp. 932-935, (1998).

[10]Emad K. A and Iman M. S, "Performance of the decorrelator receiver for DS-CDMA mobile radio system employing RAKE and diversity through Nakagami fading channel," IEEE Transactions on Communications, vol. 50, no. 10, (2002)

[11]T. Anil Kumar and K. Deerga Rao, "A new M-estimator for performance analysis of cellular digital mobile radio systems including diversity technique, IEEE Asia Pacific Conference on Circuits and Systems, (2008).

[12] Xiaodong Wang and H. V. Poor, "Robust multiuser detection in non-Gaussian channels," IEEE Transactions on Signal Processing, vol.47, no.2, pp.289-305, (1999).

[13] J.G. Proakis, Digital Communications, Fourth Edition, McGraw Hill, (2001).

[14]T. Anil Kumar, and K. Deerga Rao, " Improved Robust techniques for multiuser detection in non-Gaussian channels", Circuits Systems and Signal Processing J., vol. 25, no. 4, (2006). 